\title{
Analysis, Filtering, and Control for Takagi-Sugeno Fuzzy Models in Networked Systems
}

\author{
Sunjie Zhang, ${ }^{1}$ Zidong Wang, ${ }^{2,3}$ Jun Hu, ${ }^{4,5}$ Jinling Liang, ${ }^{3,6}$ and Fuad E. Alsaadi ${ }^{3}$ \\ ${ }^{1}$ School of Information Science and Technology, Donghua University, Shanghai 200051, China \\ ${ }^{2}$ Department of Computer Science, Brunel University, Uxbridge, Middlesex UB8 3PH, UK \\ ${ }^{3}$ Communication Systems and Networks (CSN) Research Group, Faculty of Engineering, King Abdulaziz University, \\ Jeddah 21589, Saudi Arabia \\ ${ }^{4}$ Research Institute of Intelligent Control and Systems, Harbin Institute of Technology, Harbin 150001, China \\ ${ }^{5}$ Department of Applied Mathematics, Harbin University of Science and Technology, Harbin 150080, China \\ ${ }^{6}$ Department of Mathematics, Southeast University, Nanjing 210096, China
}

Correspondence should be addressed to Sunjie Zhang; jasonzhang134@gmail.com

Received 14 July 2014; Accepted 11 August 2014

Academic Editor: Xiao He

Copyright (C) 2015 Sunjie Zhang et al. This is an open access article distributed under the Creative Commons Attribution License, which permits unrestricted use, distribution, and reproduction in any medium, provided the original work is properly cited.

\begin{abstract}
The fuzzy logic theory has been proven to be effective in dealing with various nonlinear systems and has a great success in industry applications. Among different kinds of models for fuzzy systems, the so-called Takagi-Sugeno (T-S) fuzzy model has been quite popular due to its convenient and simple dynamic structure as well as its capability of approximating any smooth nonlinear function to any specified accuracy within any compact set. In terms of such a model, the performance analysis and the design of controllers and filters play important roles in the research of fuzzy systems. In this paper, we aim to survey some recent advances on the T-S fuzzy control and filtering problems with various network-induced phenomena. The network-induced phenomena under consideration mainly include communication delays, packet dropouts, signal quantization, and randomly occurring uncertainties (ROUs). With such network-induced phenomena, the developments on T-S fuzzy control and filtering issues are reviewed in detail. In addition, some latest results on this topic are highlighted. In the end, conclusions are drawn and some possible future research directions are pointed out.
\end{abstract}

\section{Introduction}

Since its inception by Zadeh [1-3], the theory of fuzzy set and system has gone through substantial development and has been widely employed in various kinds of areas such as control engineering, signal processing, information processing, decision making, management, finance, medicine, motor industry, and robotics. As one of the most important research topics, the fuzzy logic control has been firstly proposed by Mamdani and Assilian [4,5]. Note that, in this kind of control method, the fuzzy control algorithms including a set of heuristic control rules have been utilized to achieve the desired control performance. Fuzzy sets and fuzzy logic have been used, respectively, to represent linguistic terms and evaluate the rules. More than a decade later, one of the first fuzzy filters has been provided by Vicente and Aranguren [6] where the fuzzy topological concepts have been characterized by utilizing the convergence of the designed fuzzy filter.

According to the fuzzy rules and their generation methods, the research methodologies on fuzzy control can be roughly classified into six categories [7]: traditional fuzzy control, fuzzy proportional-integral-derivative (PID) control, neurofuzzy control, fuzzy sliding mode control, adaptive fuzzy control, and fuzzy control based on Takagi-Sugeno ( $\mathrm{T}$ $S)$ model. It is worth mentioning that the T-S fuzzy model $[8,9]$ has attracted an ever-increasing research interest owing to (1) its convenient and simple dynamic structure and (2) its capability of using a set of fuzzy rules to approximate a global nonlinear system. Generally speaking, the T-S model consists of a set of local linear models which are smoothly connected by fuzzy membership functions. In this framework, the problem of T-S fuzzy control and filtering has long been 
a fascinating focus of research attracting constant attention. It is not surprising that there has been a rich body of relevant literature published in the past two decades; see, for example, [10-19] for control problems and [20-26] for filtering issues.

In recent years, because of the ever-increasing popularity of communication networks, the study of networked systems has gradually become an active research area due to the advantages of using networked media in many aspects such as low cost, simple installation, reduced weight, and power requirements, as well as high reliability [27, 28]. It is well known that the signals are often transmitted through networks which may undergo unavoidable network-induced phenomena including communication delays, packet dropouts (also called missing measurements), signal quantization, and randomly occurring uncertainties (ROUs); see [29-37] and the references therein. These network-induced phenomena would bring in particular systems complexities (e.g., abrupt structural and parametric changes) which could seriously degrade the system performance if not adequately handled in practical engineering applications. Consequently, due to the merits of approximating nonlinear systems, the T-S fuzzy models in networked environments have been introduced to describe the nonlinear networked control systems (NCSs), and the corresponding control and filtering problems for such T-S fuzzy systems with aforementioned network-induced phenomena have attracted considerable attention by many researchers during the past few years [38-41].

In this paper, we focus mainly on the control and filtering problems for T-S fuzzy systems with network-induced phenomena and aim to provide a survey on some recent advances in this area. Firstly, a variety of T-S fuzzy control and filtering issues with network-induced phenomena are discussed in great detail. Both theories and techniques for dealing with the controller or filter design are systematically reviewed. Subsequently, latest results on T-S fuzzy control/filtering problems for networked systems are surveyed and some challenging issues for future research are raised. Finally, some conclusions are drawn and several possible related research directions are pointed out.

The rest of the paper is organized as follows. In Section 2, the control and filtering problems for the T-S fuzzy systems with network-induced phenomena are reviewed. Section 3 reviews the latest results on T-S fuzzy control, filtering, and fault detection problems for networked systems and some challenging issues are highlighted at the same time. The conclusions and future work are given in Section 4.

\section{T-S Fuzzy Control and Filtering with Network-Induced Phenomena}

The signal transmission via networked systems has become prevalent and, accordingly, the network-induced issues have drawn considerable research interest. In this section, we will recall the theoretical developments of T-S fuzzy control and filtering problems from the following four aspects: communication delays, packet dropouts, signal quantization, and ROUs.
2.1. T-S Fuzzy Control and Filtering with Communication Delays. It has been well recognized that communication delays, which could be one of the causes for poor performance or even instability of the closed loop, exist universally in practical systems. In the past decades, significant research efforts have been devoted to the control and filtering problems for T-S fuzzy systems with different types of time-delays including constant time-delays, time-varying delays, multiple time-delays, infinitely distributed time-delays, Markov jumping time-delays, and interval time-delays. For instance, in [42], some new results on stability properties (asymptotical stability and input-to-state stability) have been investigated for T-S fuzzy Hopfield neural networks with constant timedelay. Furthermore, in [33], the delay-dependent stabilizability condition has been integrated with the shifted-Chebyshevseries approach and the hybrid Taguchi-genetic algorithm. An effective control scheme has been proposed to handle the quadratic finite-horizon optimal parallel distributed compensation (PDC) control problem of the T-S fuzzymodel-based time-delay systems. In order to overcome the inherent difficulty of the nonlinear optimal control issue, the corresponding design has been turned into the feasibility problem for certain linear matrix inequalities (LMIs) in a suboptimal sense that can be easily solved by means of numerically efficient convex programming algorithms.

In the context of T-S fuzzy control, in addition to the communication delays, other factors contributing to the system complexities have also been studied. For example, the parameter uncertainties are unavoidable for modeling real-world engineering systems which would result in perturbations of the elements of a system matrix [43-45]. As such, in the past decade, considerable attention has been devoted to fuzzy systems with various time-delays and parameter uncertainties, and a large number of results have been reported by exploiting the LMI approach. According to the linear differential inclusion state-space representation, a novel design scheme of fuzzy controller has been proposed in [46] to stabilize the nonlinear multiple time-delay largescale system. Moreover, the $H_{\infty}$ performance index, which is closely related to the robustness of the closed-loop system, also has been employed to evaluate the design controller. In [47], the robust $H_{\infty}$ control problem has been investigated for a class of discrete-time T-S fuzzy systems with the infinitely distributed time-delay which can be regarded as the discretization version of the infinite integral form in continuoustime case. It deserves attention that, in most of the literature mentioned above, the control problem has been considered for the case that the time-delay is not random. However, the time-delays may occur in a probabilistic method. So, stochastic time-delays over T-S fuzzy control systems also have been further researched. For instance, a T-S model has been employed to represent a networked control system with Markov jumping time-delays in [48], and the designed approach has addressed situations involving all possible network-induced delays. In [49], the probabilistic interval distribution of communication delay has been taken into account, and a robust networked controller for a class of T-S fuzzy systems has been designed, where the solvability of the networked controller design depends not only on the upper 
and lower bounds of the delay but also on its probability distribution.

Similar to the control problem with communication delays, the problem of T-S fuzzy filtering also has been attracting considerable research interests and a lot of advanced methods have been proposed to handle the network-induced time-delays. For example, the fuzzy $H_{\infty}$ filtering has been discussed in [50] for a class of nonlinear discrete-time systems with both multiple time-delays and unknown bounded disturbances. For the same kind of time-delays, in [51], a full-order $H_{\infty}$ filter has been designed to guarantee that the filtering error dynamics are stochastically stable and the given $H_{\infty}$ attenuation level is guaranteed. Additionally, it is worth mentioning that the results discussed above have been presented with delay-independent conditions. An interesting research problem is how to utilize the time-delay information to reduce the conservatism. Based on such an idea, the delay-dependent approach has been widely adopted in recent years for various time-varying delay T-S fuzzy systems. For instance, by utilizing the T-S fuzzy model, the $H_{\infty}$ filtering problem has been addressed in [52] for a class of nonlinear systems where the nonlinearities have been assumed to satisfy global Lipschitz conditions. Furthermore, a novel delaydependent piecewise Lyapunov-Krasovskii functional, which is dependent on both the upper bound of the delays and the delay interval, has been constructed in [30] to analyze the filtering error dynamics and then some sufficient conditions have been established in terms of LMIs.

Very recently, for the delay-dependent problems mentioned above, a novel technique has been provided by introducing some free-weighting matrices. The delay-dependent filter design for nonlinear systems with time-varying delay via T-S fuzzy model approach has been studied in [53], and the main technique used is the free-weighting matrix method combined with a matrix decoupling approach. The problem of delay-dependent robust $H_{\infty}$ filtering design has been investigated for a class of uncertain discrete-time statedelayed T-S fuzzy systems in [54], where the state delay has been assumed to be time-varying and of an interval-like type, meaning that both the lower and upper bounds of the timevarying delay are available. Based on the delay-dependent piecewise Lyapunov-Krasovskii functional combined with an improved free-weighting matrix method, a delay-dependent $H_{\infty}$ filter has been further designed for a class of discretetime nonlinear interconnected systems with time-varying delays via the T-S fuzzy model in [55], and it guarantees both the delay-dependent stability and the prescribed $H_{\infty}$ performance index.

\subsection{T-S Fuzzy Control and Filtering with Packet Dropouts. It} is a well-known fact that, in a networked environment, the measurement output is not consecutive but contains missing observations due to various causes such as network-induced packet loss and sensor temporal failure; see, for example, $[56,57]$. Therefore, the control problem for T-S fuzzy systems with packet dropouts has recently attracted much attention. Assume that $\delta>0$ represents a maximum allowable transfer interval. For no packet dropout case, a transmission of a packet takes place at time $t_{k}$ and the control signal will reach the plant at the instant $t_{k}+\tau_{k}$, and then the next control signal must arrive within the time interval $\left(t_{k}, t_{k}+\delta\right]$. As such, the phenomenon of packet dropout in network transmission occurs when $\tau_{k}>\delta$. According to such a fact, a guaranteed cost networked control problem has been developed for T-S fuzzy systems with both network-induced delays and packet dropouts in network transmission [19]. Recently, in [58], the fault-tolerant control approach for linear controlled plant has been extended to the case of the nonlinear networked control systems in the presence of networked-induced delay and packet dropout as well as external disturbance. By using the Lyapunov-Krasovskii functional and introducing some slack matrices, the authors have established a less conservative robust $H_{\infty}$ integrity design scheme for the T-S fuzzy systems with failures of both actuator and sensor.

Considering the unavoidable parameter uncertainties for modeling real-world engineering systems, some researchers have endeavoured to investigate fuzzy control systems with packet dropouts and parameter uncertainties. The problem of fuzzy controller design has been addressed for a class of nonlinear networked control systems approximated by uncertain networked T-S models in [59], where the timevarying network-induced delay and data packet dropout have been considered simultaneously. In [32], the robust $H_{\infty}$ control problem has been investigated for a class of uncertain discrete-time fuzzy systems with both multiple probabilistic delays and multiple missing measurements. The measurement-missing phenomenon occurs in a random way and the missing probability for each sensor satisfies a certain probabilistic distribution in the interval $[0,1]$. In [60], the problem of robust $H_{\infty}$ output-feedback control has been studied for a class of network nonlinear systems with multiple packet dropouts, where stochastic variables satisfying the Bernoulli random binary distribution are adopted to characterize the data missing phenomenon. In [61], the stochastic stability has been researched for a class of discrete-time nonlinear control systems in the T-S form with uncertain network-induced time-delays and missing measurements by using a fuzzy decentralized control approach.

In comparison to the fruitful results for control problems of T-S fuzzy systems with packet dropouts, the corresponding filtering issues have also received considerable research attention. In [62], the network-based robust fault detection problem has been studied for a class of uncertain discretetime T-S fuzzy systems with stochastic mixed time-delays and successive packet dropouts. A sequence of stochastic variables, which are mutually independent but obey the Bernoulli distribution, has been introduced to govern the random occurrences of the discrete time-delays, the distributed timedelays, and the successive packet dropouts. Moreover, if the network media are introduced to the filtering issues, the data packet dropout phenomenon, which occurs in a network environment, will naturally induce the intermittent measurements from the plant to the filter. Therefore, the problem of filter design with intermittent measurements is of significant importance and a significant amount of research effort has been made to analyze the T-S fuzzy systems with intermittent observations. The problem of $H_{\infty}$ fuzzy filtering of nonlinear systems represented by a T-S fuzzy model 
with intermittent measurements has been investigated in [63]. The measurements transmission from the plant to the filter is assumed to be imperfect and a stochastic variable satisfying the Bernoulli random binary distribution is utilized to model the phenomenon of the missing measurements, so the data packet dropout phenomenon happens intermittently. In [28], the authors have further discussed the problem of fault detection for T-S fuzzy systems with intermittent measurements. In most published papers, the measurement signal is usually assumed to be either completely missing or completely available and therefore the packet dropouts can be governed by some stochastic variables obeying the given Bernoulli distribution. However, such an assumption is quite restrictive in practice in case of fading measurements for an array of sensors. Recently, the stochastic variables with general probability distributions are adopted to characterize the data missing phenomenon in output channels. In [27], for this kind of general model, the problem of robust $H_{\infty}$ state estimation has been investigated for a class of multichannel networked nonlinear systems with both multiple packet dropouts and norm-bounded uncertainties.

\subsection{T-S Fuzzy Control and Filtering with Signal Quantization.} In networked systems, due to the application of finiteprecision arithmetic and the limited network bandwidth, quantization is an effective approach in order to reduce both the network burden and the energy consumption. However, the performance of networked systems will be inevitably subject to the effect of quantization error. As a result, it is necessary to research into (1) how the quantization phenomenon affects the system performance and (2) how to develop a suitable method to solve the addressed control and filtering problem with various network-induced phenomena involving signal quantization. It is worth noting that the problem of quantized control for nonnetworked systems has been reported as early as in 1990 [64].

So far, some preliminary results have been available in the literature; see $[36,65,66]$, for example. The problem of quantized output-feedback networked control for the continuous-time T-S fuzzy system with impulsive effects has been investigated in [66] where both the observed state and the control signals have been quantized before they were sent to the controller and the actuator. In [36], the problem of robust control has been concerned for uncertain discrete-time T-S fuzzy networked control systems with state quantization, network-induced delays, and packet dropouts. Based on the same model, by employing the fuzzy LyapunovKrasovskii functional, a less conservative delay-dependent stability condition has been derived in order to guarantee the desired stability and $H_{\infty}$ performance. For a class of continuous-time T-S fuzzy affine dynamic systems with quantized measurements, a suitable observer-based dynamic output-feedback controller has been designed by using the common/piecewise quadratic Lyapunov functions combined with both the S-procedure and the matrix inequality convexification technique in [65].

Unfortunately, the filtering problem for T-S fuzzy system with signal quantization has gained very little research attention despite its practical importance. In [67], the problem of generalized $\mathrm{H}_{2}$ filtering has been studied for a class of discrete-time T-S fuzzy systems with measurement quantization and packet loss. The quantized measurements are transmitted to the filter via an imperfect communication channel and the quantization errors are treated as sector bound uncertainties.

2.4. T-S Fuzzy Control and Filtering with ROUs. In the networked world nowadays, the parameter uncertainty serves as one of the important complexities for system modeling. The parameter uncertainties may be subject to random changes in environmental circumstances, for example, network-induced random failures and repairs of components, changing subsystem interconnections, and sudden environmental disturbances; see [34], for more details. It is worth noting that a stochastic variable obeying the given Bernoulli distribution has been utilized to characterize such a phenomenon. Such a description is more suitable for reflecting parameter variations in a random nature, particularly in the network transmission. In [34], the concept of ROUs has been firstly introduced to reflect the uncertain parameter variations and the robust sliding mode control problem has been considered for discrete time stochastic systems. By using the Lyapunov stability theory, the stability and the reachability have been investigated in detail.

Inspired by the work in [34], ROUs have been investigated for T-S fuzzy systems in recent years. For instance, the problem of robust passive control for networked fuzzy systems has been considered in [68], where ROUs, variable sampling intervals, and constant network-induced delay have been taken into account, simultaneously. In this paper, a discontinuous Lyapunov functional has been introduced for the closed-loop T-S fuzzy systems, which takes full advantage of the sawtooth structure of the time-varying interval delay induced by sample-and-hold and signal transmission. Furthermore, in [69], the $H_{\infty}$ fuzzy filtering problem has been discussed for a class of discrete-time T-S fuzzy systems with ROUs and randomly occurring interval time-varying delays as well as channel fadings. However, up until now, ROUs have not yet received adequate research attention.

\section{Latest Progress}

Very recently, the control and filtering problems for the networked T-S fuzzy systems have been intensively studied and some elegant results have been reported. In this section, we highlight some of the newest works with respect to this topic.

3.1. Bilinear T-S Fuzzy Model. In [70], the fuzzy bilinear state feedback controller based on the T-S fuzzy bilinear model has been addressed for direct current-direct current (DC-DC) converters. Via Taylor series expansion, the DCDC converters can be approximated by the bilinear model and one of this extended system's state variables is the error between the output voltage and the reference output voltage. A fuzzy bilinear state feedback controller has been designed to track the reference output voltage and it ensures that the closed-loop system is globally asymptotically stable. 
3.2. Event-Based Fuzzy Control. In [71], a discrete-time event-based communication protocol and a parallel distribution compensation controller have been appropriately codesigned so as to trade off the communication bandwidth utilization and the stability of the controlled continuous-time T-S fuzzy system. Compared with a time-triggered periodic communication scheme, the advantage of event-triggered communication scheme will not only greatly lighten the network loads but also save power of sensors. Moreover, by employing the networked T-S fuzzy model and the discrete event-triggered communication scheme, a stability criterion and a stabilization criterion about the networked T-S fuzzy system are derived in terms of matrix inequalities, and the maximum allowable delay and the feedback gain can be obtained simultaneously through solving an optimization problem. Recently, the event-triggered distributed filtering is a promising research topic.

\subsection{Fuzzy Control with Multiple Network-Induced Phenom-} ena. In [72], the $H_{\infty}$ output-feedback control problem has been addressed for a class of discrete-time fuzzy systems with randomly occurring infinite distributed delays and channel fadings. The stochastic Rice fading model has been employed to simultaneously describe the phenomena of random timedelays and channel fadings via setting different values of the channel coefficients. An $H_{\infty}$ output-feedback fuzzy controller has been designed such that the closed-loop T-S fuzzy control system is exponentially mean-square stable and the disturbance rejection attenuation is constrained to a given level by means of the $H_{\infty}$-performance index. Subsequently, sufficient conditions have been obtained for the existence of desired output-feedback controllers ensuring both the exponential mean-square stability and the prescribed $H_{\infty}$ performance. However, the phenomena of channel fadings have not yet been thoroughly studied for the T-S fuzzy system and this deserves deep investigation.

3.4. $l_{2}-l_{\infty}$ Fuzzy Filtering. In [73], the design problem of $l_{2}$ $l_{\infty}$ filters has been investigated for a class of discrete-time T-S fuzzy systems with time-varying delay. The full-order and reduced-order filters that guarantee the filtering error system to be asymptotically stable with a prescribed $H_{\infty}$ performance have been designed. By employing an inputoutput approach and a two-term approximation method, which is applied to approximate the time-varying delay, sufficient conditions have been proposed for the designed filtering error system. In this paper, the main attention has been focused on the reduction of conservativeness, such that the filter synthesis problems have feasible solutions in the fullorder and reduced-order cases. The time-varying delay in the original system has been transformed into the uncertainties so as to use the small-scale gain theorem, and then the original system has been transformed into a comparison system including two subsystems which are a constant time-delay forward subsystem and a delayed "uncertainty" feedback one.

3.5. $H_{-} / H_{\infty}$ Fault Detection. Reference [74] has been concerned with the design problem of the $H_{-} / H_{\infty}$ fault detection filter under relax conditions for the T-S fuzzy system affected by sensor faults and unknown bounded disturbances. This method has employed the technique of descriptor systems through considering sensor faults as an auxiliary state variable and a robust fault detection filter design which has the best robustness to disturbances and sensitivity to faults has been formulated. Based on nonquadratic Lyapunov functions, this design has approached the fault detection filtering problem by means of the minimization of the $H_{\infty}$ norm and the maximization of the $H_{-}$index to obtain the observer gains and the residual weighting matrix. Hence, the problems of fault detection filter with randomly occurring network-induced phenomena would be another interesting topic.

3.6. $H_{\infty}$ Filtering with Unknown Membership Functions. In [75], the $H_{\infty}$ filter has been designed for T-S fuzzy systems with unknown or partially unknown membership functions, which refer to the ones with unknown parameters or unknown perturbations or unknown variables. In this case, the designed filter over fuzzy systems, which is based on parallel distributed compensator strategy, is infeasible. In order to tackle this difficulty, a switching mechanism, which depends on the lower and upper bounds of the unknown membership functions, has been employed and further modified to construct the $H_{\infty}$ filter with varying gains. Nevertheless, in these approaches, the system states are required to be available, and thus, it is rather difficult to be used to design the $H_{\infty}$ filter.

3.7. Nonfragile $H_{\infty}$ Fuzzy Filtering. In [76], the design problem of nonfragile $H_{\infty}$ filter has been studied for continuoustime T-S fuzzy systems. The proposed filter has been assumed to have two types of multiplicative gain variations. At first, two relaxed $H_{\infty}$ filtering analysis conditions have been proposed based on useful LMIs. Next, some results have been utilized to obtain sufficient conditions for designing a nonfragile $H_{\infty}$ filter guaranteeing a $H_{\infty}$ performance of the fuzzy filtering error system. It is clear that the designed methods not only suit a standard form of the fuzzy filter but also give more relaxed design conditions through comparing them with the existing results. As a result, the analysis and design problems for the nonfragile $H_{\infty}$ filtering with randomly occurring gain variations still remain as challenging research topics.

\section{Conclusions and Future Work}

Throughout the paper, we have reviewed some recent advances on the T-S fuzzy control and filtering problems for networked systems with the network-induced phenomena. We have discussed, in great detail, various control and filtering problems for network-induced phenomena consisting of communication delays, packet dropouts, signal quantization, and ROUs. In addition, we have surveyed some latest results on control and filtering problems for T-S fuzzy systems with network-induced phenomena and pointed out some challenging issues.

Finally, based on the literature review, we provide some related directions for the future research work as follows. 
(i) The T-S fuzzy control and filtering issues for networked systems are still active research topics. In practical engineering, there still exist many more complex yet important network-induced phenomena such as channel fadings and randomly occurring gain variations, which have not yet been thoroughly studied yet. Therefore, a trend for future research is to investigate the T-S fuzzy control and filtering with more network-induced phenomena [77-81].

(ii) The problems of fault detection for T-S fuzzy systems in the presence of network-induced phenomena are of engineering significance, especially when the system is time-varying. Hence, it would be interesting to investigate the problems of fault detection filtering for time-varying T-S fuzzy systems with randomly occurring network-induced phenomena over a finite time horizon.

(iii) Note that the network-induced phenomena usually occur in a random way which makes the dynamics under consideration stochastic. In particular, the mathematical expectation and variance of random variables are two time-varying positive scalar sequences that take values on two intervals (rather than two distinctive points as in the current studies). Therefore, the control and filtering problems for TS fuzzy systems with the time-varying occurrence probability are of significant engineering importance.

(iv) Taking the energy efficiency into consideration, the T-S fuzzy control and filtering algorithms with eventtriggered mechanism are of significant engineering importance. Future works may involve the study on how to construct an exact mathematical description of event-based T-S fuzzy systems.

(v) Some other future research directions are to further investigate self-adaptive fuzzy control, nonfragile $H_{\infty}$ filtering, and multiobjective $H_{2} / H_{\infty}$ filtering problems for T-S fuzzy systems with randomly occurring network-induced phenomena.

(vi) Applications of the existing theories and methodologies to some practical engineering problems (e.g., consumer electronics, medical services, pilotless aircrafts, and industrial robots) would be another topic for future work.

\section{Conflict of Interests}

The authors declare that there is no conflict of interests regarding the publication of this paper.

\section{Acknowledgments}

This work was supported in part by the National Natural Science Foundation of China under Grants 61134009, 61329301, 11301118, and 61174136, the Natural Science Foundation of Jiangsu Province of China under Grant BK20130017, the Fundamental Research Funds for the Central Universities of China under Grant CUSF-DH-D-2013061, the Royal Society of the UK, and the Alexander von Humboldt Foundation of Germany.

\section{References}

[1] L. A. Zadeh, "Fuzzy sets," Information and Computation, vol. 8, pp. 338-353, 1965.

[2] L. A. Zadeh, "Fuzzy algorithm," Information Control, vol. 12, pp. 94-102, 1968.

[3] L. A. Zadeh, "Outline of new approach to the analysis of complex systems and decision processes," IEEE Transactions on Systems, Man, Cybernetics, vol. SMC-3, no. 1, pp. 28-44, 1973.

[4] E. H. Mamdani, "Application of fuzzy algorithms for simple dynamic plant," Proceedings of the Institution of Electrical Engineers, vol. 121, no. 12, pp. 1585-1588, 1974.

[5] E. H. Mamdani and S. Assilian, "An experiment in linguistic synthesis with a fuzzy logic controller," International Journal of Man-Machine Studies, vol. 7, no. 1, pp. 1-13, 1975.

[6] M. A. D. Vicente and M. S. Aranguren, "Fuzzy filters," Journal of Mathematical Analysis and Applications, vol. 129, no. 2, pp. 560-568, 1988.

[7] G. Feng, "A survey on analysis and design of model-based fuzzy control systems," IEEE Transactions on Fuzzy Systems, vol. 14, no. 5, pp. 676-697, 2006.

[8] T. Takagi and M. Sugeno, "Fuzzy identification of systems and its application to modelling and control," IEEE Transactions on Systems, Man, Cybernetics, vol. 15, no. 1, pp. 116-132, 1985.

[9] K. Tanaka and M. Sugeno, "Stability analysis and design of fuzzy control systems," Fuzzy Sets and Systems, vol. 45, no. 2, pp. 135156, 1992.

[10] C. N. Bhende, S. Mishra, and S. K. Jain, "TS-fuzzy-controlled active power filter for load compensation," IEEE Transactions on Power Delivery, vol. 21, no. 3, pp. 1459-1465, 2006.

[11] B. Chen, X. Liu, K. Liu, and C. Lin, "Adaptive fuzzy tracking control of nonlinear MIMO systems with time-varying delays," Fuzzy Sets and Systems, vol. 217, pp. 1-21, 2013.

[12] C.-W. Chen, "Interconnected TS fuzzy technique for nonlinear time-delay structural systems," Nonlinear Dynamics, vol. 76, no. 1, pp. 13-22, 2014.

[13] B. Ding, "Development of stability research on Takagi-Sugeno fuzzy control systems and approximation of the necessary and sufficient conditions," Fuzzy Information and Engineering, vol. 1, no. 4, pp. 367-383, 2009.

[14] S. K. Hong and R. Langari, "An LMI-based $H_{\infty}$ fuzzy control system design with TS framework," Information Sciences, vol. 123, no. 3-4, pp. 163-179, 2000.

[15] C. Hu, H. Jiang, and Z. Teng, "General impulsive control of chaotic systems based on a TS fuzzy model," Fuzzy Sets and Systems, vol. 174, pp. 66-82, 2011.

[16] C. Peng, Q.-L. Han, D. Yue, and E. Tian, "Sampled-data robust $H_{\infty}$ control for T-S fuzzy systems with time delay and uncertainties," Fuzzy Sets and Systems, vol. 179, no. 1, pp. 20-33, 2011.

[17] C. Peng, M.-R. Fei, and E. Tian, "Networked control for a class of T-S fuzzy systems with stochastic sensor faults," Fuzzy Sets and Systems, vol. 212, pp. 62-77, 2013.

[18] G. L. Serra and J. A. Silva, "Robust PID TS fuzzy control methodology based on gain and phase margins specifications," Journal of Intelligent \& Fuzzy Systems, vol. 26, no. 2, pp. 869-888, 2014. 
[19] H. Zhang, D. Yang, and T. Chai, "Guaranteed cost networked control for T-S fuzzy systems with time delays," IEEE Transactions on Systems, Man and Cybernetics Part C: Applications and Reviews, vol. 37, no. 2, pp. 160-172, 2007.

[20] W. Assawinchaichote, S. K. Nguang, and P. Shi, "Robust $H_{\infty}$ fuzzy filter design for uncertain nonlinear singularly perturbed systems with Markovian jumps: an LMI approach," Information Sciences, vol. 177, no. 7, pp. 1699-1714, 2007.

[21] J. B. Machado, R. J. G. B. Campello, and W. C. Amaral, “TakagiSugeno fuzzy models in the framework of orthonormal basis functions," IEEE Transactions on Cybernetics, vol. 43, no. 3, pp. 858-870, 2013.

[22] S. Y. Noh, J. B. Park, and Y. H. Joo, "Kalman filtering for TS fuzzy state estimation," in Proceedings of the SICE-ICASE International Joint Conference, pp. 3800-3803, Busan, Republic of Korea, October 2006.

[23] S. Noh, J. Park, and Y. Joo, "A fuzzy filter with missing measurement for observer-based T-S fuzzy models," in Proceedings of the International Conference on Control, Automation and Systems, Gyeonggi, Republic of Korea, October 2010.

[24] L. S. Saoud, F. Rahmoune, V. Tourtchine, and K. Baddari, "Generalized dynamical fuzzy model for identification and prediction," Journal of Intelligent and Fuzzy Systems, vol. 26, no. 4, pp. 1771-1785, 2014.

[25] L. Xie, C. de Souza, and M. Fu, " $H_{\infty}$ estimation for discrete time linear uncertain systems," International Journal of Robust and Nonlinear Control, vol. 1, no. 2, pp. 111-123, 1991.

[26] S. Zhou, J. Lam, and A. Xue, " $H_{\infty}$ filtering of discrete-time fuzzy systems via basis-dependent Lyapunov function approach," Fuzzy Sets and Systems, vol. 158, no. 2, pp. 180-193, 2007.

[27] J. Qiu, G. Feng, and H. Gao, "Nonsynchronized-State estimation of multichannel networked nonlinear systems with multiple packet dropouts via TS Fuzzy-Affine dynamic models," IEEE Transactions on Fuzzy Systems, vol. 19, no. 1, pp. 75-90, 2011.

[28] Y. Zhao, J. Lam, and H. Gao, "Fault detection for fuzzy systems with intermittent measurements," IEEE Transactions on Fuzzy Systems, vol. 17, no. 2, pp. 398-410, 2009.

[29] H. Ahmada and T. Namerikawa, "Extended Kalman filter-based mobile robot localization with intermittent measurements," Systems Science and Control Engineering, vol. 1, no. 1, pp. 113126, 2013.

[30] M. Chen, G. Feng, H. Ma, and G. Chen, "Delay-dependent $H_{\infty}$ filter design for discrete-time fuzzy systems with time-varying delays," IEEE Transactions on Fuzzy Systems, vol. 17, no. 3, pp. 604-616, 2009.

[31] S.-H. Chen, W.-H. Ho, J.-T. Tsai, and J.-H. Chou, "Regularity and controllability robustness of TS fuzzy descriptor systems with structured parametric uncertainties," Information Sciences, vol. 277, pp. 36-55, 2014.

[32] H. Dong, Z. Wang, D. W. C. Ho, and H. Gao, "Robust $H_{\infty}$ fuzzy output-feedback control with multiple probabilistic delays and multiple missing measurements," IEEE Transactions on Fuzzy Systems, vol. 18, no. 4, pp. 712-725, 2010.

[33] M.-R. Hsu, W.-H. Ho, and J.-H. Chou, "Stable and quadratic optimal control for TS fuzzy-model-based time-delay control systems," IEEE Transactions on Systems, Man, and Cybernetics Part A:Systems and Humans, vol. 38, no. 4, pp. 933-944, 2008.

[34] J. Hu, Z. Wang, H. Gao, and L. K. Stergioulas, "Robust sliding mode control for discrete stochastic systems with mixed time delays, randomly occurring uncertainties, and randomly occurring nonlinearities," IEEE Transactions on Industrial Electronics, vol. 59, no. 7, pp. 3008-3015, 2012.
[35] J. Liang, F. Sun, and X. Liu, "Finite-horizon $H_{\infty}$ filtering for time-varying delay systems with randomly varying nonlinearities and sensor saturations," Systems Science and Control Engineering: An Open Access Journal, vol. 2, no. 1, pp. 108-118, 2014.

[36] M. S. Mahmoud and A.-W. A. Saif, "Robust quantized approach to fuzzy networked control systems," IEEE Journal on Emerging and Selected Topics in Circuits and Systems, vol. 2, no. 1, pp. 7181, 2012.

[37] G. Wei, L. Wang, and F. Han, "A gain-scheduled approach to fault-tolerant control for discrete-time stochastic delayed systems with randomly occurring actuator faults," Systems Science and Control Engineering, vol. 1, no. 1, pp. 82-90, 2013.

[38] H. Gassara, A. El Hajjaji, M. Kchaou, and M. Chaabane, "Observer based $(Q, V, R)$ - $\alpha$-dissipative control for TS fuzzy descriptor systems with time delay," Journal of the Franklin Institute, vol. 351, no. 1, pp. 187-206, 2014.

[39] H. Gao, Y. Zhao, and T. Chen, " $H_{\infty}$ fuzzy control with missing data," in Proceedings of the IEEE International Conference on Systems, Man, and Cybernetics (SMC '07), pp. 801-806, Montreal, Canada, October 2007.

[40] S. K. Nguang, P. Shi, and S. Ding, "Fault detection for uncertain fuzzy systems: an LMI approach," IEEE Transactions on Fuzzy Systems, vol. 15, no. 6, pp. 1251-1262, 2007.

[41] X. Yang and Z. Yang, "Synchronization of TS fuzzy complex dynamical networks with time-varying impulsive delays and stochastic effects," Fuzzy Sets and Systems, vol. 235, no. 1, pp. 25-43, 2014.

[42] C. K. Ahn, "Some new results on stability of Takagi-Sugeno fuzzy Hopfield neural networks," Fuzzy Sets and Systems, vol. 179, no. 1, pp. 100-111, 2011.

[43] F. Diba, A. Arora, and E. Esmailzadeh, "Optimized robust cruise control system for an electric vehicle," Systems Science and Control Engineering, vol. 2, no. 1, pp. 175-182, 2014.

[44] C.-S. Tseng, "Robust fuzzy filter design for a class of nonlinear stochastic systems," IEEE Transactions on Fuzzy Systems, vol. 15, no. 2, pp. 261-274, 2007.

[45] X. Wang, E. E. Yaz, and J. Long, "Robust and resilient statedependent control of discrete-time nonlinear systems with general performance criteria," Systems Science and Control Engineering, vol. 2, no. 1, pp. 48-54, 2014.

[46] F.-H. Hsiao, S.-D. Xu, C.-Y. Lin, and Z.-R. Tsai, "Robustness design of fuzzy control for nonlinear multiple time-delay largescale systems via neural-network-based approach," IEEE Transactions on Systems, Man, and Cybernetics Part B: Cybernetics, vol. 38, no. 1, pp. 244-251, 2008.

[47] G. Wei, G. Feng, and Z. Wang, "Robust $H_{\infty}$ control for discrete-time fuzzy systems with infinite-distributed delays," IEEE Transactions on Fuzzy Systems, vol. 17, no. 1, pp. 224-232, 2009.

[48] Y. Zheng, H. Fang, and H. O. Wang, "Takagi-Sugeno fuzzymodel-based fault detection for networked control systems with Markov delays," IEEE Transactions on Systems, Man, and Cybernetics B: Cybernetics, vol. 36, no. 4, pp. 924-929, 2006.

[49] C. Peng and T. C. Yang, "Communication-delay-distributiondependent networked control for a class of T-S fuzzy systems," IEEE Transactions on Fuzzy Systems, vol. 18, no. 2, pp. 326-335, 2010.

[50] H. Zhang, S. Lun, and D. Liu, "Fuzzy $H_{\infty}$ filter design for a class of nonlinear discrete-time systems with multiple time delays," IEEE Transactions on Fuzzy Systems, vol. 15, no. 3, pp. 453-469, 2007. 
[51] B. Jiang, Z. Mao, and P. Shi, " $H_{\infty}$-filter design for a class of networked control systems via T-S fuzzy-model approach," IEEE Transactions on Fuzzy Systems, vol. 18, no. 1, pp. 201-208, 2010.

[52] L. Wu and Z. Wang, "Fuzzy filtering of nonlinear fuzzy stochastic systems with time-varying delay," Signal Processing, vol. 89, no. 9, pp. 1739-1753, 2009.

[53] C. Lin, Q.-G. Wang, T. H. Lee, and B. Chen, "Hinfin; filter design for nonlinear systems with time-delay through T-S fuzzy model approach," IEEE Transactions on Fuzzy Systems, vol. 16, no. 3, pp. 739-746, 2008.

[54] J. Qiu, G. Feng, and J. Yang, "A new design of delay-dependent robust $H_{\infty}$ filtering for discrete-time T-S fuzzy systems with time-varying delay," IEEE Transactions on Fuzzy Systems, vol. 17, no. 5, pp. 1044-1058, 2009.

[55] H. Zhang, H. Zhong, and C. Dang, "Delay-dependent decentralized $\mathrm{H}$ filtering for discrete-time nonlinear interconnected systems with time-varying delay based on the T-S fuzzy model," IEEE Transactions on Fuzzy Systems, vol. 20, no. 3, pp. 431-443, 2012.

[56] M. Basin, E. Sanchez, and R. Martinez-Zuniga, "Optimal linear filtering for systems with multiple state and observation delays," International Journal of Innovative Computing, Information and Control, vol. 3, no. 5, pp. 1309-1320, 2007.

[57] M. S. Mahmoud, Y. Shi, and H. N. Nounou, "Resilient observerbased control of uncertain time-delay systems," International Journal of Innovative Computing, Information and Control, vol. 3, no. 2, pp. 407-418, 2007.

[58] W. Li and D. Jiang, "Robust $H_{\infty}$ fault-tolerant control for networked control system based on Takagi-Sugeno fuzzy model," in Proceedings of the IEEE International Conference on Intelligent Computing and Intelligent Systems (ICIS '09), vol. 2, pp. 449456, Shanghai, China, November 2009.

[59] X. Jiang and Q.-L. Han, "On designing fuzzy controllers for a class of nonlinear networked control systems," IEEE Transactions on Fuzzy Systems, vol. 16, no. 4, pp. 1050-1060, 2008.

[60] J. Qiu, G. Feng, and H. Gao, "Asynchronous output-feedback control of networked nonlinear systems with multiple packet dropouts: T-S fuzzy affine model-based approach," IEEE Transactions on Fuzzy Systems, vol. 19, no. 6, pp. 1014-1030, 2011.

[61] F. Zhou, L. Liu, and G. Feng, "Fuzzy decentralized control for a class of networked control systems with time delays and missing measurements," in Proceedings of the 10th IEEE International Conference on Control and Automation (ICCA '13), pp. 306-311, Hangzhou, China, June 2013.

[62] H. Dong, Z. Wang, J. Lam, and H. Gao, "Fuzzy-model-based robust fault detection with stochastic mixed time delays and successive packet dropouts," IEEE Transactions on Systems, Man, and Cybernetics B: Cybernetics, vol. 42, no. 2, pp. 365-376, 2012.

[63] H. Gao, Y. Zhao, J. Lam, and K. Chen, " $H_{\infty}$ fuzzy filtering of nonlinear systems with intermittent measurements," IEEE Transactions on Fuzzy Systems, vol. 17, no. 2, pp. 291-300, 2009.

[64] D. F. Delchamps, "Stabilizing a linear system with quantized state feedback," IEEE Transactions on Automatic Control, vol. 35, no. 8, pp. 916-924, 1990.

[65] J. Qiu, G. Feng, and H. Gao, "Observer-based piecewise affine output feedback controller synthesis of continuous-time T-S Fuzzy affine dynamic systems using quantized measurements," IEEE Transactions on Fuzzy Systems, vol. 20, no. 6, pp. 10461062, 2012.
[66] H. Zhang, H. Yan, F. Yang, and Q. Chen, "Quantized control design for impulsive fuzzy networked systems," IEEE Transactions on Fuzzy Systems, vol. 19, no. 6, pp. 1153-1162, 2011.

[67] C. Zhang, G. Feng, and J. Qiu, "Generalized $H_{2}$ filter design for T-S fuzzy systems with quantization and packet loss," in Proceedings of the IEEE Symposium on Computational Intelligence in Control and Automation, pp. 52-59, Paris, France, April 2011.

[68] Z.-G. Wu, P. Shi, H. Su, and J. Chu, "Network-based robust passive control for fuzzy systems with randomly occurring uncertainties," IEEE Transactions on Fuzzy Systems, vol. 21, no. 5, pp. 966-971, 2013.

[69] S. Zhang, Z. Wang, D. Ding, and H. Shu, "Fuzzy filtering with randomly occurring parameter uncertainties, interval delays, and channel fadings," IEEE Transactions on Cybernetics, vol. 44, no. 3, pp. 406-417, 2014.

[70] J. Baek and M. Park, "Fuzzy bilinear state feedback control design based on TS fuzzy bilinear model for DC-DC converters," International Journal of Electrical Power \& Energy Systems, vol. 42, no. 1, pp. 710-720, 2012.

[71] C. Peng, Q.-L. Han, and D. Yue, "To transmit or not to transmit: a discrete event-triggered communication scheme for networked takagi-sugeno fuzzy systems," IEEE Transactions on Fuzzy Systems, vol. 21, no. 1, pp. 164-170, 2013.

[72] S. Zhang, Z. Wang, D. Ding, and H. Shu, " $H_{\infty}$ fuzzy control with randomly occurring infinite distributed delays and channel fadings," IEEE Transactions on Fuzzy Systems, vol. 22, no. 1, pp. 189-200, 2014.

[73] X. Su, P. Shi, L. Wu, and Y.-D. Song, "A novel approach to filter design for T-S fuzzy discrete-time systems with time-varying delay," IEEE Transactions on Fuzzy Systems, vol. 20, no. 6, pp. 1114-1129, 2012.

[74] M. Chadli, A. Abdo, and S. X. Ding, " $H_{-} / H_{\infty}$ fault detection filter design for discrete-time Takagi-Sugeno fuzzy system," Automatica, vol. 49, no. 7, pp. 1996-2005, 2013.

[75] X.-J. Li and G.-H. Yang, "Switching-type $H_{\infty}$ filter design for T-S fuzzy systems with unknown or partially unknown membership functions," IEEE Transactions on Fuzzy Systems, vol. 21, no. 2, pp. 385-392, 2013.

[76] X.-H. Chang and G.-H. Yang, "Nonfragile $H_{\infty}$ filter design for T-S fuzzy systems in standard form," IEEE Transactions on Industrial Electronics, vol. 61, no. 7, pp. 3448-3458, 2014.

[77] M. Darouach and H. Souley Ali, "Optimal unbiased functional filtering in the frequency domain," Systems Science and Control Engineering, vol. 2, no. 1, pp. 308-315, 2014.

[78] G. Kumar and K. Kumar, "Network security—an updated perspective," Systems Science and Control Engineering, vol. 2, no. 1, pp. 325-334, 2014.

[79] L. Qin, X. He, and D. H. Zhou, "A survey of fault diagnosis for swarm systems," Systems Science and Control Engineering, vol. 2, no. 1, pp. 13-23, 2014.

[80] Q. Wang, H. Gao, F. Alsaadi, and T. Hayat, "An overview of consensus problems in constrained multi-agent coordination," Systems Science and Control Engineering: An Open Access Journal, vol. 2, no. 1, pp. 275-284, 2014.

[81] X. Zhang and L. Yang, "A fiber Bragg grating quasi-distributed sensing network with a wavelength-tunable chaotic fiber laser," Systems Science and Control Engineering, vol. 2, no. 1, pp. 268274, 2014. 


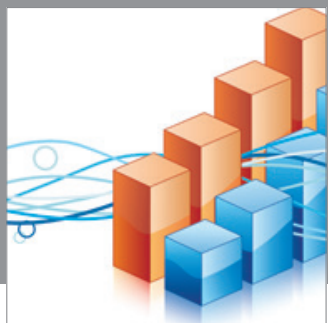

Advances in

Operations Research

mansans

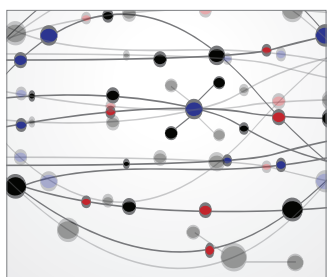

The Scientific World Journal
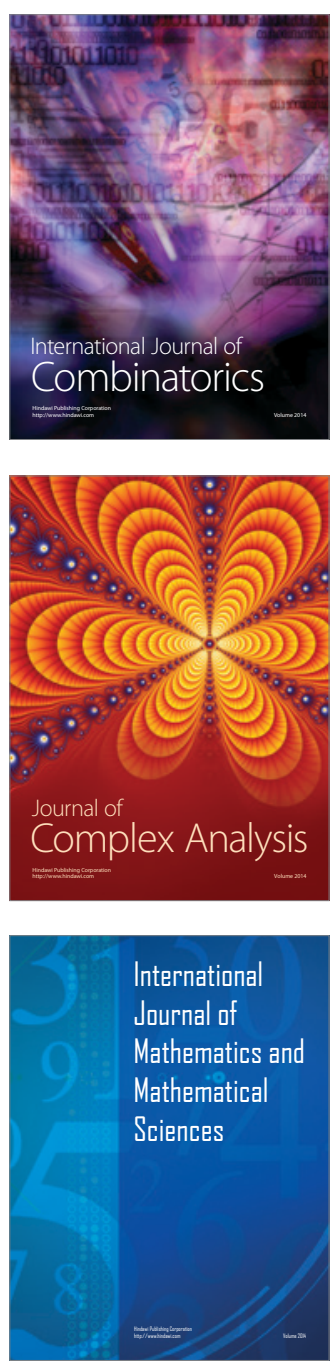
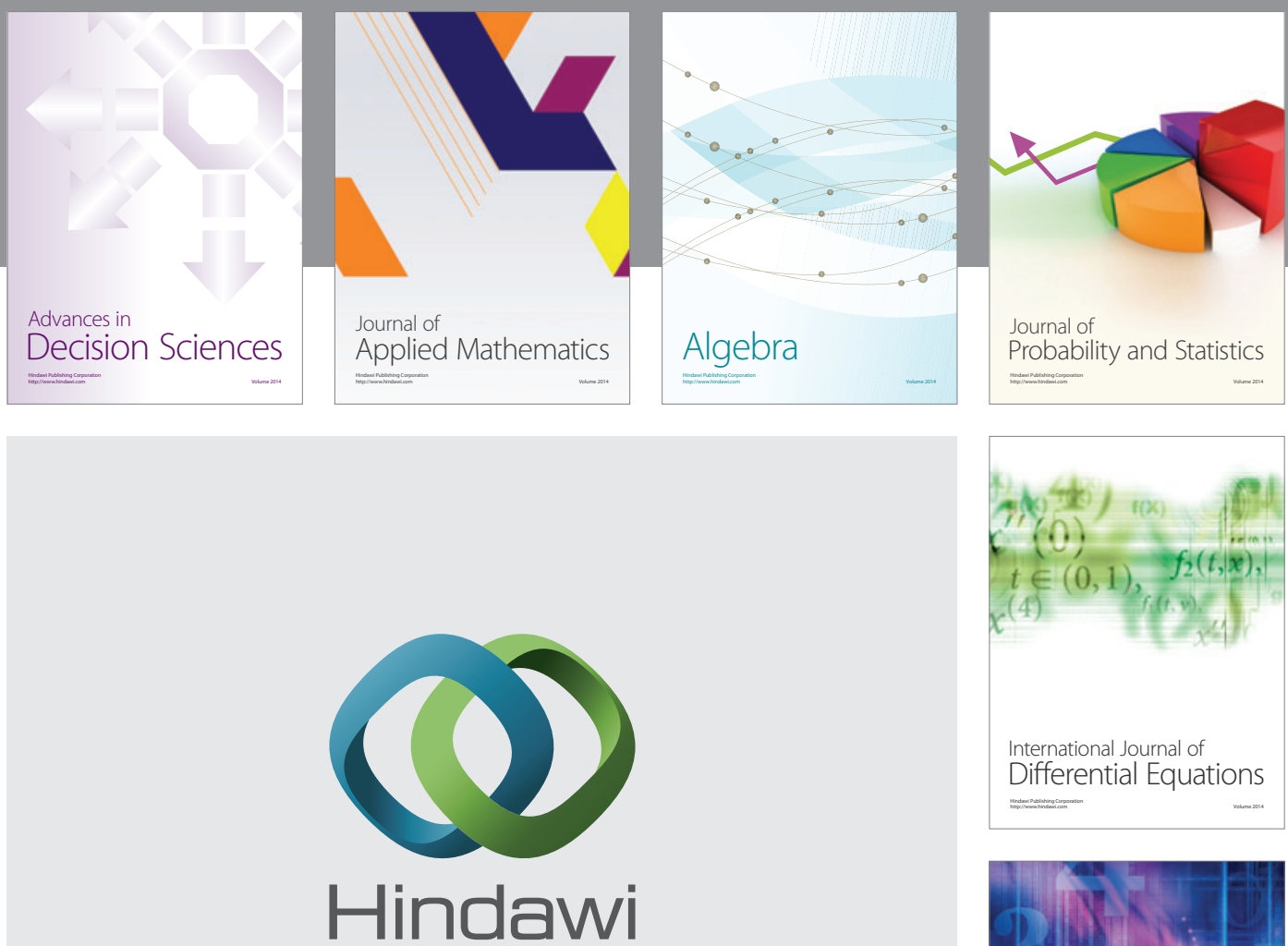

Submit your manuscripts at http://www.hindawi.com
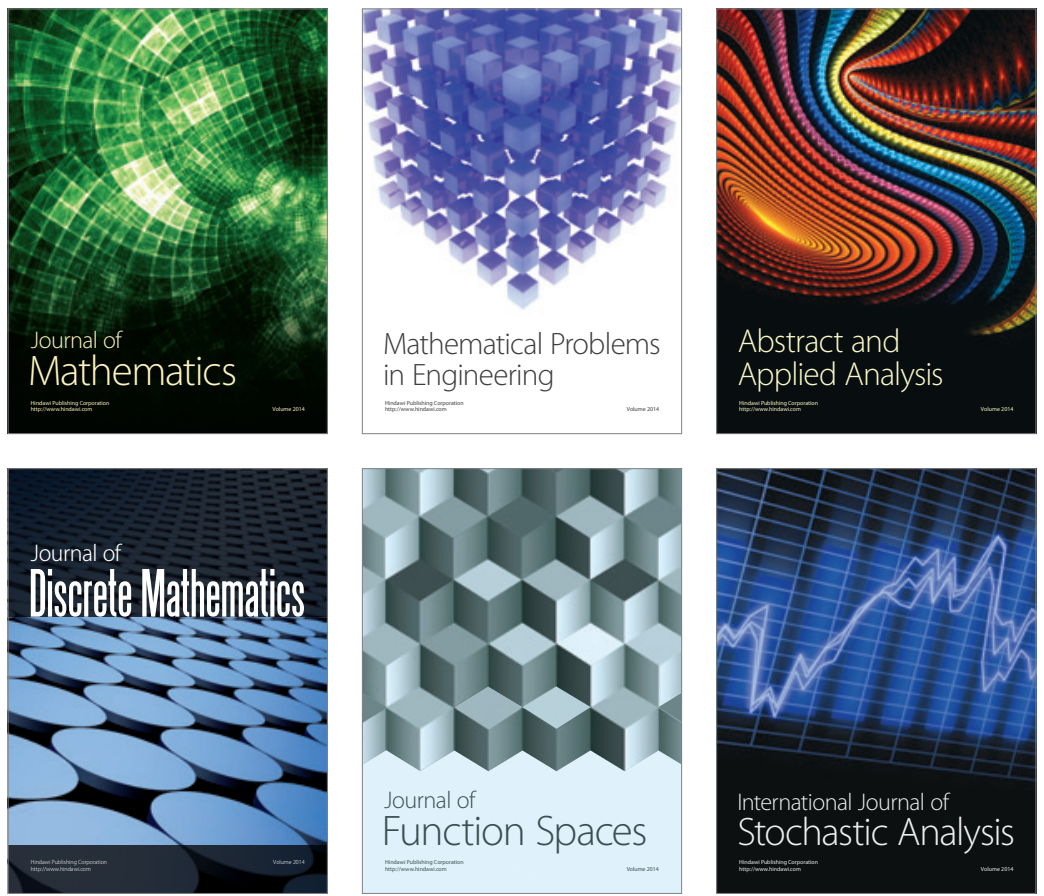

Journal of

Function Spaces

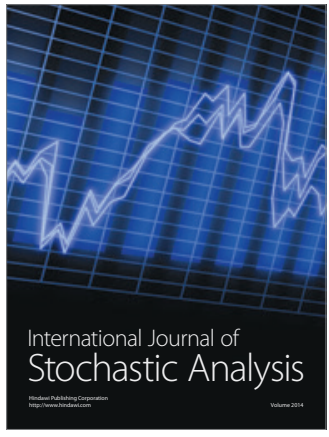

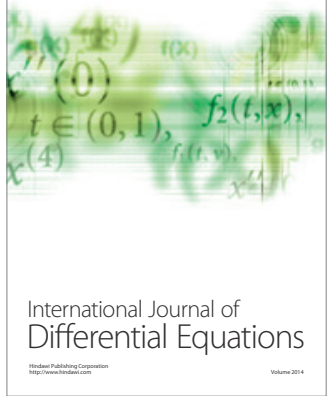
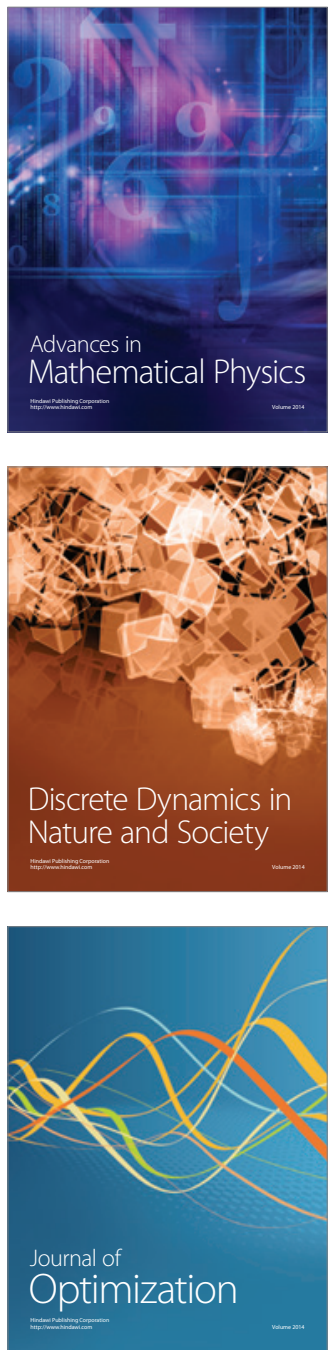